\title{
O Army Method e o desenvolvimento da Linguística Aplicada nos Estados Unidos ${ }^{1}$
}

\section{The Army Method and the development of Applied Linguistics in the United States}

José Marcelo Luna*

Universidade do Vale do Itajaí - UNIVALLI

Florianópolis - Santa Catarina / Brasil

RESUMO: Este artigo deriva de pesquisa historiográfica sobre o ensino de português nos Estados Unidos durante os anos 40, 50 e 60 do século 20. Baseado em fontes primárias, como livros, períódicos e jornais da época, o trabalho apresenta objetivamente a relação do Army Specialized Training Program, que se tornou conhecido como Army Method, com o desenvolvimento da Linguística Aplicada ao ensino de línguas estrangeiras nos Estados Unidos.

PALAVRAS-CHAVE: Historiografia, Linguística Aplicada, Ensino de línguas, Army method, Estados Unidos.

ABSTRACT: This article derives from a historiographic research on the teaching of Portuguese in the United States during 1940 through 1960. Based on primary sources like books, periodicals and newspapers of the time, the work presents objectively the relationship between the Army Specialized Training Program, also known as the Army Method, and the development of Applied Linguistics to the teaching of foreign languages in the United States.

KEYWORDS: Historiography, Applied Linguistics, Language teaching, Army method, United States.

\section{Considerações iniciais}

Embora ainda seja abordada como uma disciplina científica nova, a Historiografia da Linguística - HL - institucionaliza-se muito rapidamente em universidades e centros de pesquisa de todo o mundo, em particular na Inglaterra, na França, no Canadá e nos Estados Unidos.

\footnotetext{
${ }^{1}$ Trabalho derivado de pesquisa de pós-doutorado, com o apoio da Capes, durante o período de 2010/2011 na Universidade do Texas, em Austin.

*mluna@univali.br
} 
A HL apresenta-se para a comunidade científica com o objetivo de descrever e explicar como se adquiriu, produziu, formulou e desenvolveu o conhecimento linguístico em um determinado contexto (SWIGGERS, 1990). Essa proposta de trabalho estrutura-se com base no pressuposto de que o conhecimento linguístico é construído por meio de um processo de interação dinâmica entre os precursores, seguidores e também opositores de um referido conhecimento.

Para dar conta da reconstrução das práticas linguísticas passadas, a HL explora, com base em materiais informacionais publicados e marginais, a dimensão pessoal, que se relaciona com o papel dos agentes no processo de desenvolvimento do conhecimento, bem como a dimensão social, a qual identifica o contexto e as suas influências sobre o conhecimento linguístico.

Com essa abordagem, a importância do estudo historiográfico se evidencia para os profissionais da linguagem e para a própria Linguística. Segundo Koerner (1989), o conhecimento histórico acerca de sua disciplina é o que caracteriza um "verdadeiro cientista". Ao conhecer a origem dos pressupostos teóricos e dos métodos, bem como suas limitações, o pesquisador habilita-se a lidar com problemas imprevistos e com mudanças de interpretaçôes, e a reconhecer os avanços reais, no seu campo, de suas variações ao longo do tempo. Sobre a contribuição do trabalho historiográfico, Brekle (1986), por sua vez, revela:

In this sense the reason for a historiography of science is simply the attempt to keep available the knowledge which has once been accumulated, to communicate to later generations of linguists what earlier generations have thought about linguistic problems in the broadest sense, or what attempts at solutions they have made (1986, p. 5).

Assim motivados, vimos, há mais de uma década, imprimindo uma perspectiva historiográfica aos nossos estudos sobre línguas estrangeiras. Em particular, o ensino de português nos Estados Unidos destaca-se como nosso interesse por razões que, a partir de uma perspectiva historiográfica, podem estar interrelacionadas. Referimo-nos ao movimento, quase desconhecido no Brasil, de mais de três séculos e meio de implantação do Português como Língua Estrangeira - PLE - naquele país, marcados, nos termos de alguns pesquisadores, "por altos e baixos". Referimo-nos, paralelamente, à tradição americana na área de Linguística Aplicada - LA - ao ensino de línguas, e à formação e ao desenvolvimento de grupos e associações de especialistas em PLE em algumas universidades dos Estados Unidos. 
Apesar de representar um relevante objeto de pesquisa para a Linguística, além de uma fonte valiosa de conhecimento sobre a natureza e o status da língua portuguesa e da identidade e cultura brasileiras no mundo, a historiografia do ensino do português no exterior ainda se encontra em estágio de produção incipiente.

Objetivando contribuir com o desenvolvimento dessa área, desenvolvemos, como parte integrante de um estágio pós-doutoral / Capes com a Universidade do Texas em Austin, uma pesquisa historiográfica sobre o ensino de português nos Estados Unidos durante as décadas de 40 a 60 do século 20.

No presente artigo, apresentamos os resultados consolidados referentes ao anos de 40, tendo como foco a relação do Army Specialized Training Program - ASTP, que se tornou conhecido como Army Method e o desenvolvimento da Linguística Aplicada ao ensino de línguas estrangeiras nos Estados Unidos. Trata-se de um levantamento, a partir de fontes primárias, como livros, artigos de periódicos e de jornais da época, do contexto socioeconômico e político-estratégico, bem como das concepções linguísticas que caracterizam a época.

\section{A segunda guerra mundial e o chamado Army Method}

Com o advento da Segunda Guerra, o quadro de irregularidade de oferta de cursos de línguas estrangeiras começa a mudar em instituiçōes de ensino dos Estados Unidos. A sociedade e as instituiçõos americanas, que tinham, durante a Primeira Guerra Mundial, desmerecido a língua e a cultura do inimigo e repudiado qualquer coisa que pertencesse a ele, passa, na Segunda Guerra, a querer aprender a língua dos povos com cujos países os Estados Unidos estavam envolvidos, fosse como inimigo, fosse como aliado. Objetivamente, a motivação era militar e estratégica, mas também política e econômica; visava ao período da guerra em si, mas também ao que viria depois, por exemplo, as oportunidades de negócios.

É assim que o Brasil, por exemplo, começa a ser visto e referido nas mais diversas publicações do período, não só naquelas especializadas em ensino de línguas e educação como também em negócios e política externa.

Since the outbreak of the present war in 1939 the situation has changed radically. With Lisbon the only important European Atlantic seaport in neutral territory, Portugal began to loom large in the daily news. More important has been the growing interest, caused by the 
war, in all the Latin-American countries and the consequent realization of the importance of Brazil (MILLER, 1942, p. 351).

Embora a importância da variante continental fosse reconhecida pela relação com a vasta e tradicional literatura produzida em Portugal, a escolha pela variante do português do Brasil começa, nos anos 40, a ser expressa e formalmente feita. É o que se depreende também de Miller:

Although Brazil has yet to produce a literature comparable to that of Portugal, the political and economic importance of Brazil to this country seems to favor our adoption of the Brazilian pronunciation (1942, p. 353).

Influenciando esse processo, qual seja, a escolha por uma variante e pela relacionada abordagem de ensino, estava a noção de utilidade e funcionalidade da língua para a comunicação oral. Essa noção, que se desenha a partir de princípios apresentados pelos trabalhos de Franz Boas, Edward Sapir e Leonard Bloomfield, serviu de base para o ASTP, que se tornaria conhecido como o Army Method.

O clima de opiniāo da Segunda Guerra Mundial, marcado pelas necessidades de comunicação oral com os povos nela direta e indiretamente envolvidos, fez alguns linguistas reconhecerem o princípio da primazia da língua oral, bem como desenvolverem estratégias metodológicas para o ensino de línguas estrangeiras.

Em relatório de 8 de maio de 1943 sobre um dos cursos intensivos de português dados pelo Instituto do American Council of Learned Societies, instituição apontada como fomentadora e promotora da maioria dos cursos de português nos Estados Unidos entre os anos 30 e 40, Zeitlin (1943) deixa clara a relação do ASTP com as experiências didático-pedagógicas que se desejariam naquela e nas três décadas seguintes com o ensino de português nos Estados Unidos. Descrevendo alguns aspectos desse curso, ela destaca:

Um segundo aspecto consiste em nós termos feito um esfôrço para reproduzir até onde nos foi possível o ambiente brasileiro: [...] Quanto à parte oral do curso, também pensámos sempre na língua como um instrumento de que os alunos precisavam para conversar com brasileiros no Brasil. [...] procurámos ensinar-lhes os vocábulos e giros necessários para as situações em que pudessem encontrar-se no Brasil - não só na estação de estrada de ferro como também o museu, da biblioteca, do arquivo, etc. (ZEITLIN, 1943, p. 48). 
Para caracterizarmos a década de 40 , é também digna de nota a realização, ao longo dos anos de 1944 e 1945, das Conferences on the Teaching of Spanish and Portuguese. Promovidas pela National Education Association, com recursos do Office of the Coordinator of Inter-American Affairs, essas conferências foram feitas em vários estados do país, tendo como objetivo a discussão de temas como:

The place of the Hispanic Languages and languages in general in training for world citizenship; the objectives of their teaching; applications to civilian classes of the Army experience with language classes; new teaching materials; Spanish in the elementary school; and preparation of foreign language teachers (NICHOLS, 1945, p. 130).

Os temas anunciados e tratados nessa verdadeira série de conferências podem ser tomados como reveladores da importância que o ensino de línguas estava tendo para a sociedade americana e do lugar do chamado Army Method na agenda e no trabalho dos professores de línguas estrangeiras nos Estados Unidos.

Embora o ASTP não possa ser tomado como uma abordagem nova em termos de princípios teóricos, ele representa uma comprovada inovação e uma forte influência, pelas estratégicas metodológicas nele empregadas, para o ensino de línguas em geral.

A entrada dos Estados Unidos na Segunda Guerra Mundial significou a constatação de que a sociedade americana não tinha conhecimento suficiente de línguas estrangeiras; nunca antes, para o americano, tinha sido tão importante se comunicar com tantas pessoas de línguas tão diferentes do inglês.

Quando as Forças Armadas buscaram, nos seus quadros, as pessoas com conhecimento de outras línguas, depararam com a seguinte situação: soldados e oficiais que ou não tinham estudado língua estrangeira alguma na vida escolar ou que, embora tivessem, não conseguiam usá-las proficientemente, em especial na sua modalidade oral.

A competência de produção e de compreensão orais era pelos militares entendida como a condição necessária para interagir sobre os mais diversos assuntos do cotidiano, o que exigiria também conhecimento da região e dos povos, ou seja, conhecimento cultural. A constatação da deficiência das Forças Armadas americanas nesse sentido e a abordagem que o programa consequentemente tomaria podem ser encontradas em Matthew: 
Early in the war - in fact, before Pearl Harbor - the armed services were aware of the need for men with a working knowledge of the languages and peoples of many regions into which it was possible that the course of the war would take them $(1947$, p. 3).

Para dar conta desses objetivos, as Forças Armadas se valem, como já sugerido, de alguns dos princípios da Linguística, em particular o da primazia da língua falada. É nos estudos de Boas, Sapir e Bloomfield, desenvolvidos entre os anos 20 e 30, que os militares reconhecem os fundamentos para as estratégias metodológicas que viriam desenvolver e tornar conhecidas para a sociedade em geral.

Pela necessidade de formar o maior número de soldados e oficiais com domínio de uma ou mais línguas estrangeiras no mais curto período de tempo possível, as Forças Armadas entendiam que a situação de ensino teria que ser intensiva e de quase imersão. Nesse sentido, eles aproveitaram experiências práticas do American Council of Learned Societies, que tinha desenvolvido, em 1943, um programa intensivo de ensino das línguas "pouco conhecidas" nos Estados Unidos.

As características metodológicas do ASTP são variadas. A primeira delas e a que mais causaria discussão quando das tentativas de aplicação às escolas em geral diz respeito à carga-horária. Em vez de três ou quatro horas semanais, o Programa, em tese, se desenvolvia em vinte horas por semana. Isso siginificava dedicação quase que integral aos estudos da língua estrangeira.

Outra característica da abordagem diz respeito ao lugar da gramática nas aulas. Para todos os níveis, mas especialmente para os iniciantes, o ensino da gramática só era recomendado como auxílio no processo de imitação do padrão da língua estrangeira. Pela ênfase na língua falada, linguistas e professores do ASTP preconizaram que a produção e a compreensão escritas não interferissem na aprendizagem da fala, devendo ser introduzidas apenas depois de assegurado o controle da oralidade.

Seguindo o princípio da primazia da fala, os alunos eram estimulados a produzir e compreender oralmente, da forma mais fluente e precisa possível. Para tanto, aplicava-se o conhecimento derivado da Fonética, que, desde o século 19, já despontava como uma base científica para o ensino de línguas (LUNA, 2000). Nesse sentido, a estratégia metodológica básica era a imitação do falante nativo, chamado de informante, o que se fazia por sessóes de 'pattern drills'. A repetição da pronúncia padrão até a memorização iria justificar a utilização de recursos didáticos mecânicos, especialmente aqueles comuns aos laboratórios de línguas. 
Trata-se da aceitação, expressa e materializada, da concepção de língua como um sistema de hábitos, e do aprendizado como um processo de condicionamento. Os linguistas e professores responsáveis pelo ASTP e todos aqueles que seriam por essa prática influenciados nos anos que se seguiriam não só aceitam essa caracterização do fenômeno linguístico como procedente como a tornam a sustentação do seu método. A resposta automática, que seria característica do comportamento linguístico na língua materna de um aluno, passa o ser o objetivo maior da sala de aula de língua estrangeira pela abordagem chamada de Army Method.

Esse objetivo justificaria a diversidade e a quantidade de drills. Para os linguistas e professores responsáveis pelo ASTP, essas sessōes de exercícios tipicamente são, ao mesmo tempo, a expressão da crença nos princípios da linguística contrastiva. De fato, os exercícios do tipo drill eram especialmente desenhados para tomar três quintos do total de uma aula e, assim, garantir a ênfase nas formas e estruturas da língua estrangeira em contraste com as formas e estruturas da língua materna do aluno.

Os exercícios não somente vão enfatizar as estruturas constrastantes, mas, ao fazê-lo, vão combater a interferência do sistema da língua materna sobre aquele da língua em estudo. Essa concepção de língua e o seu desdobramento em estratégias metodológicas podem ser tomados como expressão da influência da Linguística em sua natureza aplicada ao ensino de línguas estrangeiras nos Estados Unidos.

Not only did ASTP help to devise new methods of teaching a foreign language (involving intensive work utilizing drill and informants), but it also showed that language teaching could and should be coordinated with the teaching of the various disciplines concerned with a given area of the world. The ASTP was the first major application of the science of linguistics to language teaching. Most important of all, perhaps, the ASTP units helped to develop the personnel for the work the universities were to do at the war's end (AXELROD; BIGELOW, 1962, p. 8).

Foi exatamente com o objetivo de determinar a influência do ASTP no ensino de línguas de instituições do país que o American Council on Education desenvolveu um estudo em 1947. De forma geral, os resultados desse trabalho dão conta de que o Army Method havia inspirado algumas mudanças nas instituições de ensino. 
A primeira das descobertas foi que os Colleges estavam oferecendo cursos intensivos de línguas para iniciantes, isto é, encontros de mais horas por semana; nessas aulas, o estudo revela, os alunos estavam sendo expostos a um número de drills incomparavelmente maior do que nas aulas chamadas tradicionais, aquelas orientadas pela abordagem para a leitura.

O uso de drills e de outras atividades de produção e compreensão orais nas aulas de línguas estrangeiras das instituiçōes escolares americanas evidencia a ruptura com o chamado método da leitura e a consequente adoção da abordagem audiolingual. Embora a literatura da década de 40 se refira quase que exclusivamente à abordagem como Army Method, o princípio da primazia da fala, sobre o qual se apoiam as estratégias de ensino no contexto militar e nas instituições civis dos Estados Unidos, é o mesmo que norteia a abordagem audiolingual.

A pesquisa do American Council on Education consegue também demonstrar que algumas universidades começam, a partir de término da Segunda Guerra Mundial, a oferecer línguas consideradas raras ou pouco conhecidas nos Estados Unidos. Matthew (1947), a propósito, relaciona esses cursos, entre os quais se encontra o de língua portuguesa:

The world-wide scale on which this enterprise was established is best shown by an enumeration of the languages that were studied, especially if one bears in mind the related area programs which was to accompany the study of the language. These languages, some of which had never been taught before in American universities, were: Annamese, Arabic [...], Bengali, Bulgarian, Burmese, Chinese [...], Czech, Dutch, Finnish, French, German, Greek, Hindustani, Hungarian, Italian, Japanese, Korean, Malayan, Norwegian, Persian, Polish, Portuguese, Russian, Serbo-Croatian, Spanish, Swedish, Thai, and Turkish (MATTHEW, 1947, p. 4).

Por fim, mas não menos importante para este trabalho, o estudo do American Council on Education revela que cursos de cultura e de civilização começam a aparecer nos currículos de algumas universidades. Registra-se, assim, a criação de "majors" em uma determinada "área".

É, de fato, com a motivação das Forças Armadas que os estudos de área se desenvolvem nos Estados Unidos. O conhecimento sobre a geografia, a história, os costumes, enfim, a cultura da região e do país em que os soldados e oficiais americanos teriam de atuar foi reconhecido como absolutamente complementar ao conhecimento da língua estrangeira. 
Matthew (1947) relata que o argumento básico para a inclusão dos estudos de área no ASTP foi a referência a um relatório de um oficial encarregado de um programa de governo militar na Alemanha durante a Primeira Guerra Mundial. Nesse documento, pode-se encontrar uma descrição do grau de desconhecimento dos soldados americanos em relação não só à língua mas também à região em que estavam atuando. Sobre as implicações e deliberaçôes desse relatório, ele acrescenta:

This report convinced the military and civil officials that special training for officers who were assigned to military government duty would be required if there was to be an improvement over conditions prevailing in the last war. These conditions revealed not only lack of training - for there was no training - but also considerable misunderstanding of the problems confronting such an officer, as well as total inability to cope with the language or to understand the people and their environment (MATTHEW, 1947, p. 54).

Se comparada às poucas experiências documentadas com o estudo de áreas nas universidades até os anos 40, a diferença conceitual básica do ASTP é a sua ênfase nos estudos culturais de forma integrada aos estudos da língua estrangeira. A relação entre língua e cultura é assim formalmente reconhecida, sendo ensinada como uma unidade de estudo. Essa integração pode ser vista como, possivelmente, uma real inovação da abordagem dos militares. Isso porque, até então, as experiências de ensino de língua e cultura desenvolvidas por algumas universidades americanas são caracterizadas como programas de estudos regionais, não integrados de forma intensiva a uma língua.

These prewar area programs presented knowledge of a cultural region in terms of competing academic disciplines, each discipline scheduled separately and in sequence rather than as an element of an integrated and synthesized course. It is to the credit of the wartime area programs, and of those who planned them, that area study was established on an integrated basis and that it was spread over a wide area of disciplines covering the contemporaneous scene (MATHEW, 1947, p. xiii).

Essa noção de unidade entre cultura e língua e de uma abordagem integrada para o estudo de línguas estrangeiras foi reforçada ao longo dos anos que se seguiram, muito em razão dos avanços e da socialização de estudos na área da Linguística. As pesquisas com as chamadas línguas vivas, em sua modalidade falada, subsidiaram o entendimento de que a língua não existe 
independentemente ou em um vácuo. Em vez disso, ela integra o padrão de comportamento geral de um povo e, o que é mais fundamental para o ensino de componentes culturais, a língua torna-se um instrumento de comunicação mais efetivo quando os alunos são expostos ao maior número possível de informações sobre os costumes e os comportamentos dos povos cujas línguas eles estão aprendendo.

Os Centros de Língua e de Área das universidades americanas, criados ou desenvolvidos com os recursos do National Defense Education Act$\mathrm{NDEA}^{2}$ - a partir do início dos anos 60 , organizaram os seus currículos, valorizando a indissociabilidade entre a língua e a cultura. Isso se manifestou pontualmente na elaboração de materiais didáticos que incluíam e destacavam os aspectos culturais de regiốes e de países.

\section{O "recrutamento" de linguistas e o desenvolvimento da linguistica aplicada}

Como vimos, a Segunda Guerra Mundial fez gerar a preocupação com o falta de conhecimento de línguas estrangeiras dos militares americanos, fato que determina o desenvolvimento do Army Method. A partir do que se pode chamar de uma verdadeira publicização dos seus bons resultados na preparação de alunos para falar e compreender uma língua estrangeira em situaçôes reais do dia a dia, podemos dizer, parafraseando Parker (1961), que os Estados Unidos descobriam o Army Method.

A adaptação do método a uma situação diferenciada daquela das forças armadas passa a ser objeto de uma série de artigos, publicados em diversas revistas especializadas em educação. Mesmo aqueles autores absolutamente simpáticos ao uso do método nas escolas em geral não iriam desconsiderar as características originais do Army Method. Para produzir os resultados propagados (aqui não nos cabe avaliar a legitimidade desse fato em si), os requisitos tomados pelas escolas militares eram, segundo Parker (1961), os seguintes:

(1) Ample time - hour for hour the Army's "9 months" were equivalent to 6 years of high school language study; (2) very small classes; (3) superlatively trained teachers - frequently two instructors

\footnotetext{
${ }^{2}$ Assinada em 2 de setembro de 1958, esta lei federal foi instituída para fomentar o desenvolvimento da educação nos Estados Unidos, com provisões específicas para o ensino de línguas estrangeiras nas escolas e universidades americanas.
} 
per class, one a native speaker; (4) up-to-date equipment; (5) students with a high IQ and with a strong motivation for language study; and, finally, (6) concentration on language study to the exclusion of everything else (PARKER, 1961, p. 91).

Nos anos que se seguiram, muitos especialistas vão tomar o ASTP e a década de 40 como motivaçôes para trabalhos sobre a natureza aplicada da Linguística. O "recrutamento" de linguistas para planejar e preparar o material didático de que precisavam as Forças Armadas dos Estados Unidos durante a Segunda Guerra Mundial parece determinar e evidenciar o papel da linguística para o professor de línguas em geral e de estrangeiras em particular.

A década de 40 é por isso referida como marco de aproximação entre a teoria e a prática. Em artigo sugestivamente intitulado "Linguistics and the Language Teacher", Hanzeli (1968) destaca:

Over the last twenty years, linguistics and language teaching have formed such strong bonds that thoroughgoing change in one must have some repercussion in the other. [...] our story begins in 1941, with the intensive language programs of the American Council of Learned Societies, converted, two years later, into the wartime Army Specialized Training Programs. These and similar programs instituted by the other armed forces mobilized the small number of professional linguists then available and involved them in the urgent task of language teaching (HANZELI, 1968, p. 42-43).

O envolvimento de linguistas no ASTP é, como já mencionamos, considerado como uma expressão da natureza aplicada da Linguística. $\mathrm{O}$ Programa é assim visto pela implicação direta que se previa à época e que efetivamente teve no ensino de línguas estrangeiras para a sociedade de forma geral. Dito de outra forma, as estratégias de ensino que se configurariam como Army Method não foram desenvolvidas originalmente tendo como único alvo a preparação de militares para a Guerra.

A revisão da literatura pertinente a esse aspecto nos fornece evidência de que os professores, militares e civis, além dos linguistas envolvidos no ASTP reconhecem que os programas de língua e de área das Forças Armadas desenvolveram certos aspectos da teoria educacional, há muito sustentados por educadores civis, que seriam absolutamente aplicáveis à demanda e à clientela da sociedade em geral. Em Matthew (1947), podemos encontrar referências a alguns trabalhos acadêmicos que tratam da aplicação do método em escolas 
e universidades dos Estados Unidos. Além dos reveladores títulos, (abaixo citamos apenas quatro de algumas dezenas de artigos semelhantes) merecem destaque as datas de suas produções:

Agard, Frederick B. "Language Lessons War Has Taught", Virginia Journal of Education, XXXVIII (February 1945), 230-42.

"Army Methods in Foreign Languages Adapted for Civilian Students", School and Society, LIX (May, 6, 1944), 324-25.

Bushnell, Marjorie R. "The Army Technique in the High School Class”, Modern Language Journal, XXVIII (February 1944), 190-91.

Carmody, Francis J. "ASTP Gives No Help to French Teachers", California Journal of Secondary Education, XX (May 1945), 257-62. (encontrado em Matthew, 1947)

A grande maioria desses trabalhos, como podemos depreender dessa citação, foi produzida durante o período da Segunda Guerra Mundial, tendo todos sido publicados imediatamente após o armistício em revistas educacionais, entre elas algumas da área de ensino de línguas. Os títulos, as datas de publicação e os seus meios de divulgação permitem-nos concluir que a concepção das estratégias de ensino, configurada como Army Mehod, não fora de algo exclusivo para as forças armadas.

Pelo envolvimento de linguistas e o consequente reconhecimento de antecedentes dos princípios teórico-metodológicos comuns à abordagem audiolingual, o que se chamou de Army Method tem seus fundamentos em teorias e práticas bem distantes do período da Guerra. Contudo, esse evento não perde por isso a sua importância como fator motivador para o ensino e a aprendizagem de línguas e como catalisador de esforços e recursos para o desenvolvimento dessa prática. Matthew (1947), a propósito, afirma:

The story of the Army and Navy foreign language and area programs, which are characterized by the close relationship between area and language, is one of the captivating interest (MATTHEW, 1947, p. xii).

A catalização que destacamos é a apresentaçao da linguística em sua natureza aplicada ao ensino de línguas. A década de 40 é inquestionavelmente aquela que reúne a maior parte de estudos sobre o lugar da ciência da linguagem na sala de aula do professor de línguas. Subsidiando essa nossa afirmação, Ferguson (1966) avalia a referida década, dizendo: 
When linguists and linguistics began to move significantly into the field of language teaching in the United States in the nineteen forties, certain aspects of the linguists' knowledge about language and their attitude towards it came to be regarded as basic principles in any application of linguistics to language teaching (FERGUSON, 1966, p. 52).

Nos termos de Ferguson, um dos princípios básicos acordados por especialistas e a população americana em geral é que a língua falada precisaria ser priorizada. $\mathrm{O}$ autor associa essa compreensão e decisão diretamente ao evento da Guerra, na década de 40. A visão do linguista de primazia da fala, adquirida décadas antes por estudos embasados na Psicologia e na Fonética, coincide com o interesse nacional amplamente caracterizado e divulgado de competência oral durante o conflito e nos anos que se seguiriam.

Como já pensamos ter deixado claro, falar a língua estrangeira destacavase como necessidade de militares nos territórios ocupados e/ou aliados e como motivação para fazer negócios e explorar oportunidades de emprego nos Estados Unidos e no exterior no chamado período pós-guerra.

A "coincidência" da visão do princípio da primazia da fala com o interesse nacional repercute diretamente na defesa e preconização da abordagem audiolingual para o ensino de línguas, o que, pelo envolvimento dos linguistas, ficaria também conhecido como método linguistico. Isso se faria durante os anos 40 e, pelo menos, durante as duas décadas seguintes. No relatório de 1962 da Northeast Conference on the Teaching of Foreign Languages, seu editor William F. Bottiglia, em artigo sugestivamente intitulado "Linguistics and Language Teaching", reclama:

We must emphasize certain things which linguistics is not. Teachers of foreign languages often think that linguistics is a way of learning the languages themselves, or a method of language teaching (BOTTIGLIA, 1962, p. 4).

De fato, um ano antes de Bottiglia assim se manifestar nessa de uma série de conferências sobre o ensino de línguas nos Estados Unidos, William G. Moulton publica um memorável artigo sobre a natureza aplicada da Linguística. Intitulado "Applied Linguistics in the Classroom", e publicado na PMLA - Publications of the Modern Language Association of America, este trabalho deriva de uma palestra dada pelo autor no General Meeting on the Foreign Language Program na Philadelfia, em 1960. Nela, Moulton também trata de esclarecer a relação da Linguística com as abordagens ou os métodos de ensino de línguas estrangeiras: 
There has been far too much loose talk recently about the application of linguistics to language teaching, [...] . There are those who talk of the "linguistic method" of language teaching, as if to oppose it to some hypothetical "non-linguistic" method used in the benighted past. [...] Linguistics is not a teaching method, but a growing body of knowledge and theory (MOULTON, 1961, p. 1).

O objetivo de esclarecer os limites da Linguística Aplicada para resolver os problemas existentes na sala de aula de línguas é comum a maior parte dos estudos e publicações do início dos anos 60. Caracterizado como tinha ficado o problema do ensino de línguas estrangeiras nos Estados Unidos no período pré- e pós-guerra, e vinculado como acabou ficando o método audiolingual à Linguística, a década de 60 começa com cobranças e defesas da aplicabilidade da ciência da linguagem.

Repercutindo esse clima de opinão, marcado e determinado particularmente pelo NDEA, então recém assinado, a Modern Language AssociationMLA - anuncia, no dia 16 de fevereiro de 1959, o estabelecimento de um Centro de Linguística Aplicada - CAL. Sediado em Washington, e apoiado finaceiramente pela Ford Foundation, teve como primeiro diretor Charles A. Ferguson. Na primeira edição do seu peródico - The Linguistic Reporter, em abril de 1959, o seu objetivo é expressamente anunciado:

[The Center] will serve as a clearing house for universities, government agencies, and other institutions or individuals concerned with the application of linguistic science to practical language problems (1959, p. 1).

O objetivo do CAL é pelo próprio Ferguson (1967) lembrado oito anos depois, novamente em artigo do The Linguistic Reporter:

The fact was clear, however, as the idea of the center was taking shape in 1957-58, that not even nineteenth-century findings were being applied to the solution of language problems, let alone the existing advances of the current research (FERGUSON, 1967, p. 1).

Ao fazer essa introdução ao que seria uma avaliação dos primeiros oito anos do CAL, Ferguson (1967) nos dá base para afirmar que havia o reconhecimento, em meio aos linguistas dos períodos aqui mais diretamente abordados, das origens dos princípios. Em outras palavras, pode-se entender que o século 19 havia denunciado o problema de ensino de línguas e reclamado 
mudanças, embasadas cientificamente, para a sua prática (LUNA, 2000). O fato de não terem sido absorvidos os princípios e aplicados em sala de aula pode ser explicado pela conjunção de fatores extra-linguísticos que agem sobre uma dada prática pedalinguística.

A propósito, falando sobre uma das que seriam as implicaçôes da Linguística para o ensino de línguas - o reconhecimento de que a língua é antes de tudo falada - Bottiglia sugere ser esse um aspecto há muito conhecido como válido e importante, mas pouco efetivamente aplicado, o que justificaria a ênfase no seu uso no ensino:

This has been repeated many times by linguistic analysts, and in fact is in danger of becoming a boring truism. Nevertheless, no matter how many times we repeat it, and even recognize its truth from an abstract point of view, we still often find it difficult to apply in practice (BOTTIGLIA, 1962, p. 5).

Objetivamente, a aplicação prática da Linguística é apresentada, também para dizer sobre o papel do então recém criado CAL, pelo seu Diretor Adjunto, Morgan Raleigh Jr.. Depois de mencionar que a Linguística tem aplicações em áreas que hoje poderíamos relacionar com a fonoaudiologia, estilística, antropologia e alfabetização / letramento, Raleigh afirma que a aplicação mais saliente da ciência da linguagem é ao ensino de línguas estrangeiras. Nesse sentido, ele relaciona o que já seriam as contribuições da Linguística até a criação do Centro, cabendo a este vê-las difundidas e evidenciadas nas mais diversas práticas pedalinguísticas do país.

It is our sincere hope that cooperative efforts between linguistics and language teachers will continue to foster an increasing application of the findings of linguistics to the field of language teaching (RALEIGH, 1959, p. 5).

Resumidamente, Raleigh (1959) vocaliza a concepção linguística e a convicção a respeito da abordagem para o ensino de línguas divididas pelos linguistas e professores diretamente envolvidos com esse trabalho nos Estados Unidos. Como se pode depreender da citação abaixo, ele destaca a orientação estruturalista e, assim, práticas de valorização da abordagem audiolingual e contrastiva da língua. 
how language functions (language learning is a skill and is acquired like other skills, such as learning to swim or play tennis); the importance of the oral-aural approach (it is generally agreed that the basic point of departure is the spoken language ...); and, most important of all, the teaching materials should be based on comparative analysis of both the target language and the native tongue of the learner (RALEIGH, 1959, p. 4).

Para bem caracterizamos o desenvolvimento da Linguística Aplicada nos Estados Unidos, convém expandir um pouco mais a referência a respeito dos estudos contrastivos. Para tanto, valemo-nos, mais uma vez, de Ferguson em sua avaliação dos primeiros oito anos do CAL, publicada em 1967. Nesse importante artigo, Ferguson afirma que o CAL, ao longo do período citado, tratou de desenvolver estudos que não tinham sido ou não poderiam ser assumidos pelas universidades. Ele fornece como exemplos quatro grandes projetos, sendo a Série de Estudos Contrastivos a que mais diretamente se relaciona com o que estamos aqui tratando.

A Constrastive Structure Series caracteriza-se como uma série de estudos contrastando as estruturas fonológicas e gramaticais do inglês e das cinco línguas mais comumente ensinadas, à época, nos Estados Unidos, quais foram, o Francês, o Alemão, o Italiano, o Russo e o Espanhol. A produção de estudos contrastivos e a disponibilização dos seus resultados na forma de livros significariam a expressão maior de coerência com os princípios da Linguística Descritiva, e com os objetivos do CAL. Convém lembrar que, ao se propor contribuir com a solução de problemas de ensino de línguas, o Centro o fazia com as convicções sustentadas pelos linguistas da época.

\section{Considerações finais}

A descrição e a explicação do contexto em que se desenvolveram o chamado Army Method e a Linguística Aplicada nos Estados Unidos foram feitas a partir de publicações, como livros e, principalmente, artigos de periódicos e jornais da época. Escritos por professores e linguistas, hoje reconhecidos como grandes nomes da Linguística norte-americana, esses documentos apresentam volume e consistência suficientes para serem tomados como expressão do clima de opinião da época.

Seguindo o método para o trabalho historiográfico, podemos evidenciar a rede de fatores socioeconômicos e político estratégicos que influenciaram na promoção de uma abordagem para o ensino de línguas estrangeiras. A Segunda 
Guerra Mundial serve, em outras palavras, como um catalisador ao trazer, para os debates e ações relacionados à educação linguística dos militares americanos e da sociedade em geral, estudiosos e professores de línguas estrangeiras dos Estados Unidos. Embora esses profissionais, principalmente aqueles que se percebiam como linguistas, já reconhecessem a natureza aplicada da ciência da linguagem, é comprovadamente sob o clima de opinião favorável ao ensino de línguas nos Estados Unidos que a Linguística Aplicada começa a se desenvolver.

Essas considerações levam-nos a fazer referência às palavras de Kelly, em seu clássico de 1969, 25 Centuries of Language Teaching. Para ele, os princípios teóricos disponíveis para o ensino de línguas não sofreram mudanças significativas ao longo de toda história. As aparentes mudanças dizem respeito aos meios e recursos usados, a partir dos princípios, para a definição de métodos. $\mathrm{O}$ que sofre uma mudança constante é a forma como os princípios e os procedimentos são apresentados a uma geração. Consequentemente, vista sob a ótica da rede de fatores que constituem a manifestação de um conhecimento sobre linguagem e sobre as línguas, a razão para o surgimento de uma ideia, para a sua utilização, bem como para o seu abandono e ressurgimento só pode ser encontrada na sociedade em que essa manifestação foi desenvolvida.

\section{Referências bibliográficas}

AXELROD, J.; BIGELOW, D. N. Resources for Language and Area Studies. Washington, D.C: American Council on Education, 1962.

BOTTIGLIA, W. F. Linguistics and Language Teaching. Reports of the Working Committees, 1962 Northeast Conference on the Teaching of Foreign Languages, p. 3-17, 1962.

BREKLE, H. E. What is the History of Linguistics and to what end is it studied? A Didactic Approach. In: BYNON, T.; PALMER, F. R. (Ed.). Studies in the History of Western Linguistics. Cambridge: Cambridge University Press, 1986.

FERGUSON, C. A. The Role of the Center for Applied Linguistics: 1959-1967. The Linguistic Reporter, v. 9, n. 6, p. 1-5, 1967.

FERGUSON, C. A. Applied Linguistics. Language Teaching: Broader Contexts. Northeast Conference on the Teaching of Foreign Languages. Reports of the Working Committee, p. 50-58, 1966.

HANZELI, V. E. Linguistics and the Language Teacher. Foreign Language Annals, v. 2, n. 1, p. 42-50, 1968. 
KELLY, L. G. 25 Centuries of Language Teaching. Rowley: Newbury House, 1969.

KOERNER, K. Models in Linguistic Historiography. In: KOERNER, K. (Ed.). Practicing linguistic historiography: selected essays. Amsterdam: John Benjamins Publishing Company, 1989.

LUNA, J. M. F. O Português na Escola Alemã de Blumenau: da formação à extinção de uma prática. Blumenau: Edifurb, 2000.

MATTHEW, R. J. Language and Area Studies in the Armed Services. Their Future Significance. Washington, D.C: American Council of Education, 1947.

MLA and Ford Announce New Center. The Linguistic Reporter, v. 1, n. 1, p. 1, 1959.

MOULTON, W. G. Applied Linguistics in the Classroom. PMLA Publications of The Modern Language Association of America, v. LXXVI, n. 2, p. 1-6, 1961.

MILlER, C. The Place of Portuguese in American Education. Education a Monthly Magazine, v. 62, n. 6, p. 351-353, 1942.

NICHOLS, M. W. The History of Spanish and Portuguese Teaching in the United States. In: DOYLE, H. (Org.). A Handbook on the Teaching of Spanish and Portuguese With Special Reference to Latin America. Boston: D.C. Heath and Company, 1945. p. 99-146.

PARKER, W. R. The National Interest and Foreign Languages: Washington: U.S. Govt Print Off, 1961.

RALEIGH, M. Jr. Linguistics and Language Teaching. The Linguistic Reporter, v.1, p. 4-5, 1959.

SWIGGERS, P. Histoire et Historiographie de l'enseignement du français: modèles, objets et analyses. Ėtudes de Linguistique Appliquée, v. 78, p. 27-44, 1990. ZEITLIN, M. A. O Ensino de Português nos Estados Unidos. Modern Language Forum, v. XXVIII, n. 3-4, p. 39-49, 1943. 